\title{
sciendo
}

10.2478/aucft-2020-0012

\section{PHYTOCHEMICAL COMPOSITION OF BREWERS' SPENT GRAINS}

\author{
- Short communication -
Kristina IVANOVA*, Ivan BAKALOV*, Vesela SHOPSKA**, Peter NEDYALKOV**, Georgi $\operatorname{KOSTOV}^{* * 1}$
* Institute of Food Preservation and Quality, Plovdiv, Agricultural Academy, 154 Vasil Aprilov Boulevard, 4003, Plovdiv, Bulgaria
** Department of Wine and Beer Technology, University of Food Technologies, Plovdiv, 26 Maritsa Boulevard, 4002, Plovdiv, Bulgaria

\begin{abstract}
Brewers' spent grains (BSG) are the main waste product of the brewing industry, accounting for about $85 \%$ of the total waste materials. Their composition is different (due to various brewing technologies) but includes high levels of dietary fiber, proteins, essential amino acids, polyphenols, antioxidants, vitamins and fats. The aim of the present study was to investigate the phenolic content and antioxidant activity (the radical scavenging assay (DPPH) and ferric reducing antioxidant power (FRAP)) of 19 different malt types and their BSG. The highest phenolic content was demonstrated by the BSG of Caraaroma malt, whereas the BSG of Carafa Special type 1 had the highest DPPH radical scavenging activity and ferric reducing antioxidant power. Based on the results obtained, malts with the highest biological value were selected for inclusion in the composition of functional foods.
\end{abstract}

Keywords: malt, brewers' spent grains, FRAP, DPPH, selection

\section{INTRODUCTION}

Brewers' spent grains (BSG) form about $85 \%$ of the waste products in the brewing industry. They are an intermediate product of beer production, and their quantity and quality composition allow their inclusion in functional foods (Aliyu and Bala, 2011; Mussatto et al., 2006).

The amount of BSG and their composition depend on the mashing method and mashing regime used to produce the beer type. The mash lautering method is equally important for the qualitative and quantitative composition of the BSG. New filtration systems are known to reduce the amount of phenolic compounds in the BSG because the phenolic compounds pass into the wort under the higher processing pressure (Mussatto et al., 2006).

BSG are a lignocellulosic material that contains certain amounts of cellulose, non-cellulosic polysaccharides and lignin. Since beer is currently produced all year round, they are a raw material that is always available. Until now, the main application of spent grains has been restricted to animal feed.

Received: 19.12.2019.

Accepted in revised form: 20.04.2020
There are limited applications of BSG as a substrate for biotechnological processes (Aliyu and Bala, 2011; Mussatto et al., 2006).

BSG have their place in human nutrition on account of their high protein, fiber and essential amino acid content. A review of the specialized literature shows that this waste product is used in a number of nonfood industries, but it is important that as a food waste product, it is automatically included in the group of permitted additives for food production (Mussato and Roberto, 2006). BSG also provide dietary fiber and polyphenols which can improve the quality and biological value of food products (Mussatto et al., 2008; Aliyu and Bala, 2011).

Barley and barley malt are excellent sources of phenolic components with antioxidant and radicalscavenging properties (Wannenmacher et al., 2018; Dvořáková et al., 2008). Data show that malt brans contain about $212.85 \mathrm{mg}$ of gallic acid equivalent (GAE)/kg of phenolic compounds (Farcas et al., 2014).

In addition to being an additive in animal feed, BSG can also be successfully incorporated into human foods, such as bread and biscuits, to increase their

${ }^{1}$ Corresponding author. E-Mail address: george kostov2@abv.bg 
fiber content. As a result, these foods are thought to lead to the prevention of some socially significant diseases. An increase in the fiber content was achieved when $30 \%$ of BSG was incorporated into wheat flour for the production of enriched dietary bread (Hassona, 1993; Miranda et al., 1994a,b; Ozturk et al., 2002; Mussatto et al., 2006). Ozturk et al. (2002) reported that BSG with different particle sizes (small $<212 \mu \mathrm{m}$; medium 212-425 $\mu \mathrm{m}$; large $425-850 \mu \mathrm{m})$ were introduced into wheat flour for biscuit production in 5 to $25 \%$ amounts. The results showed an increase in the dietary fiber content proportional to the size of the malt particles, which indicated the potential of this waste product from the brewing industry.

The aim of the present work was to investigate the phenolic content and the antioxidant potential of different types of BSG derived from malt used for the production of different types of beer.

(Kalcheva-Karadzhova et al., 2016; Singleton and Rossi, 1963) with some modifications described in Ivanova et al., 2017. The results obtained were expressed as $\mathrm{mg} \mathrm{GAE} / \mathrm{dm}^{3}$.

The following malt types were used in the presen work: Pils, Vienna, Wheat, and Pale Ale (Best Malt, Germany); Carafa Special 1 (CS1), Vienna, Melanoidin, Carapils, Munich 1, Caramunich 1, Caramunich 2, Carared, Pilsner, Carahell, Caraaroma, Carafa Special 2 (CS2), Smoked Rauch, Special Weiss, and Rye (Wayermann, Germany).

\section{Mashing method}

Congress mashing of all malts was conducted in a Lochner LB-8 according to Analytica EBC, 2007. The resulting mash was filtered through MachereyNagel MN 614 1/4 diameter $320 \mathrm{~mm}$ filter paper (Analytica EBC, 2007; Ivanova et al., 2017). The BSG were dried at $70^{\circ} \mathrm{C}$ to a constant weight (for their preservation).

Determination of total polyphenol content (TPC) The total polyphenol content was determined according to the method of Singleton and Rossi
Antioxidant activity according to the ferric
reducing antioxidant power (FRAP) assay

The ferric reducing antioxidant power was measured by the method of Benzie and Strain (Kalcheva-Karadzhova et al., 2016; Benzie and Strain, 1996) with some modifications described in Ivanova et al., 2017. The results were expressed as $\mu \mathrm{mol}$ Trolox equivalents (TE)/100g.

\section{Antioxidant activity according to the 2,2- diphenyl-1-picrylhydrazyl (DPPH) assay}

The radical scavenging ability was measured by the method of Brand-Williams et al. 1995 (KalchevaKaradzhova et al., 2016; Brand-Williams et al., 1995) with some modifications described in Ivanova et al., 2017. The results were expressed as $\mu \mathrm{mol} \mathrm{TE} / 100 \mathrm{~g}$.

\section{RESULTS AND DISCUSSION}

\section{Malt phenolic content and antioxidant capacity} The present study was conducted with 19 different malt types used in breweries in the Republic of Bulgaria. For convenience in the interpretation of the results, the malts were divided into two main groups: basic malt types and special malt types. The basic malt types were the malts whose amount was more than $50 \%$ of the malt mixture for beer production. The special malts participated in a smaller percentage in the malt mixture and provided a certain color and/or aroma to the wort. Another reason for the separation of the malts and BSG was the fact that the two malt groups underwent different degrees of treatment, resulting in a different phenolic content and antioxidant activity.

All malts showed normal brewing characteristics. The results on the phenolic content determined by
Singleton and Rossi's method as well as their antioxidant capacity (determined by DPPH and FRAP) are presented in Table 1.

The basic malt types were characterized by a relatively low phenolic compound content, which increased with the increase in malt color. The highest phenolic content was exhibited by the Special Weiss malt followed by the Munich 1 malt. The low phenolic compound content was characteristic of the basic malt types since phenolic compounds were responsible for the beer colloidal stability, and it was therefore necessary to use malt with a low phenolic compound content.

The basic malt types were characterized by approximately similar content in terms of their biological value. The highest levels of DPPH radical scavenging and ferric reducing ability were reported 
for the malts with the increased processing rate: Special Weiss and Munich 1. Similarly to the phenolic compound content, the malt antioxidant capacity increased with the increase in malt color. In the group of special malts, the trends described in the increase in phenolic content and antioxidant activity determined by the DPPH and FRAP methods were maintained. These indicators increased with the increase in the degree of malt processing, i.e. with the enhancement of its color. The increase in the antioxidant potential of special malts could also be explained by the presence of high melanoidine concentrations. The high antioxidant activity of special malt species was due not only to the phenolic compounds but also to the high concentrations of melanoidins and reductants formed during the malt kilning and/or roasting (Carvalho et al., 2016). Only the functional Caraaroma and Smoked Rauch malt types were an exception to these trends. They are used in brewing to provide a certain type of beer flavor; therefore, their processing is similar to the production of basic malt types.

Table 1. Phenolic content and antioxidant capacity of different malt types

\begin{tabular}{|c|c|c|c|c|c|c|}
\hline & \multirow[b]{2}{*}{ Malt type } & \multirow[b]{2}{*}{ Producer } & Color** & TPC & DPPH & FRAP \\
\hline & & & EBC units & $\begin{array}{c}\mathrm{mg} \\
\mathrm{GAE} / \mathrm{dm}^{3}\end{array}$ & \multicolumn{2}{|c|}{$\mu \mathrm{mol} \mathrm{TE} / 100 \mathrm{~g}$} \\
\hline \multicolumn{7}{|c|}{ Basic malts* } \\
\hline $1 * * *$ & Pils & Best Malt & 3.30 & 235.64 & 296.50 & 401.46 \\
\hline 13 & Pilsner & Weyermann & 3.20 & 269.52 & 229.58 & 783.13 \\
\hline 2 & Vienna & Weyermann & 8.50 & 257.58 & 434.91 & 609.79 \\
\hline 6 & Vienna & Best Malt & 7.75 & 309.01 & 1768.86 & 801.46 \\
\hline 18 & Special Weiss & Weyermann & 8.10 & 1837.58 & 5392.00 & 2521.00 \\
\hline 3 & Wheat & Beat Malt & 8.10 & 166.15 & 681.45 & 257.29 \\
\hline 5 & Pale Ale & Best Malt & 8.18 & 296.26 & 1609.84 & 753.96 \\
\hline 9 & Munich 1 & Weyermann & 8.79 & 1266.15 & 1528.00 & 5426.46 \\
\hline \multicolumn{7}{|c|}{ Special and fuctional malts } \\
\hline 4 & CS 1 & Weyermann & 900.00 & 842.68 & 4287.00 & 2834.00 \\
\hline 7 & Melanoidin & Weyermann & 70.00 & 409.01 & 2009.23 & 1518.13 \\
\hline 8 & Carapils & Weyermann & 4.50 & 332.48 & 1644.97 & 728.96 \\
\hline 10 & Caramunich 1 & Weyermann & 90.00 & 1837.58 & 1578.00 & 7718.13 \\
\hline 11 & Caramunich 2 & Weyermann & 120.00 & 2082.48 & 16486.70 & 7218.13 \\
\hline 12 & Carared & Weyermann & 50.00 & 1949.83 & 1500.00 & 6426.00 \\
\hline 14 & Carahell & Weyermann & 25.00 & 2041.66 & 1119.00 & 5466.00 \\
\hline 15 & Caraaroma & Weyermann & 400.00 & 12.48 & 155.99 & 71.04 \\
\hline 16 & CS 2 & Weyermann & 1150.00 & 2551.87 & 3590.00 & 16051.46 \\
\hline 17 & Smoked Rauch & Weyermann & 6.00 & 11.77 & 137.22 & 803.13 \\
\hline 19 & Rye & Weyermann & 7.00 & 241.66 & 224.04 & 484.79 \\
\hline
\end{tabular}

* the results on the basic malt types are cited according to (Shopska et al., 2019);

** according to https://www.weyermann.de/ and https://bestmalz.de/en/

*** the numbers in column 1 show the order of malts in figures 1,2 , and 3

The results obtained are consistent with those cited in the work of Moreira et al., 2013 and McCarthy et al., 2012, which report differences in phenolic compounds in BSG. The differences observed were due to the barley type used to produce malt, on the one hand, and the different extraction conditions used by the researchers, on the other (Dvoráková et al., 2008; Magalhães et al., 2011). In addition, the difference might be due to melanoidins formed during the Maillard reaction during the kilning process. Melanodins are known to have antioxidant capacity, but there are also studies proving that they can retain polyphenols in their structure, which is why the total content of free polyphenols decreases (Inns et al., 2011; Maillard and Berset, 1995). The observed differences from the studies of other authors may also be explained by the approach used to extract the phenolic compounds from the malts and BSG (Meneses et al., 2013).

\section{Phenolic content and antioxidant potential of brewers' spent grains}

Brewers' spent grains are a waste product of wort production and as such, they can be used to make functional foods as they contain various components, such as phenolic compounds and melanoidins. The results on the phenolic compound content and their antioxidant potential are shown in figures 1 to 3 .

Data on the content of total phenolic compounds indicate that between 50 and $90 \%$ of the phenolic compounds passed into the wort. Exceptions were 
found in some of the special malt types where an increased phenolic compound content in the BSG was observed. Data in the literature indicate that the residual phenolic compound content in BSG is due to the inactivation of enzymes responsible for their hydrolysis from the cell walls. This deactivation occurs at temperatures above $45^{\circ} \mathrm{C}$ (Wannemacher et al., 2018).

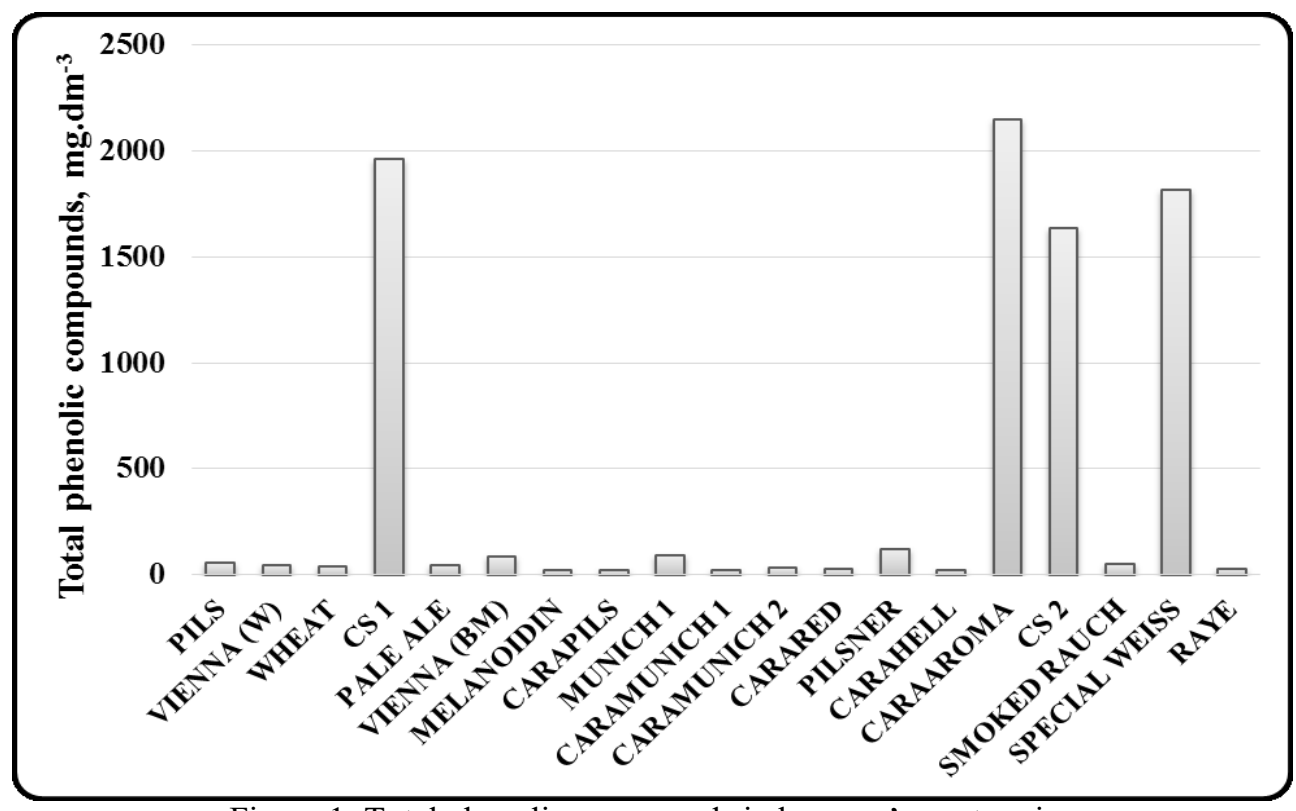

Figure 1: Total phenolic compounds in brewers' spent grains

In some malts, an increased phenolic compound content in BSG compared to the original amount in the respective malt type was reported. Two explanations can be offered. First, the method takes into account not only the phenolic content but also some of the coloring components. The second probable explanation relates to the release of some of the compounds from the matrix in which they are detained, but also their inability to pass into the wort.

The phenolic compound profile is important for the amount of phenolic compounds in spent grains. Mainly phenolic acids pass into the wort (Shopska et al., 2019; Szwajgier, 2009).

The degree of malt roasting is also important for the BSG phenolic content. Malt roasting causes both degradation of phenolic compounds and polymerization; hence, the profile of phenolic compounds in spent grains differs from that of malt and wort.

As can be seen from Figure 1, the amount of phenolic compounds in the BSG of the special malt was higher but it did not differ significantly from that of the basic malt types. Compared to the data cited in Shopska et al., 2019, there were some differences. We can attribute these differences not only to the malt type used but also to the production modes used by the manufacturers of the respective malt type. However, we can assume that the BSG obtained from special malt types have a higher phenolic content than basic malt types.
The radical system used to determine the antioxidant capacity requires the application of at least two evaluation indicators. In the present study, the evaluation of the antioxidant capacity was made on the basis of two indicators: scavenging of the DPPH radical (Figure 2) and ferric reducing ability by FRAP (Figure 3). This, together with the phenolic profile of each of the malts and the corresponding $\mathrm{BSG}$, can be used to describe the biological potential of each sample.

The results in Figure 2 and Figure 3 show that there was a correlation between the phenolic compound content, the malt processing degree and the antioxidant potential of the BSG. The highest antioxidant capacity was determined for the Caramunich 2 malt: approximately $16500 \mu \mathrm{mol}$ $\mathrm{TE} / 100 \mathrm{~g}$ (Table 1), and for the Carafa Special type 1 (CS1) BSG: $2250 \mu \mathrm{mol} \mathrm{TE} \mathrm{/} \mathrm{100g} \mathrm{(Figure} \mathrm{3).}$

In most of the variants tested, the ferric reducing ability was higher than that of the DPPH radical scavenging, which should be explained by the antioxidant profile of the spent grain components (Table 1, Figure 2 and Figure 3). The high antioxidant activity of some malt species resulted in a significant amount of melanoidins that were formed during the drying and baking of the malt. It is a known fact that melanoidins have antioxidant capacity and this potential increases with the degree of malt processing. 
Selection of BSG with a high biological potential and possibilities for their application in functional foods

The data from the study of the different types of malts and BSG indicated that the BSG had a high biological potential. The results obtained showed that the special BSG had a wider range of phenolic compounds and antioxidant components, and it is therefore appropriate to use the BSG obtained from the production of red or dark beers in functional foods. Another potential BSG source are craft breweries that produce different types of beer, hence BSG derived from many different types of malt.

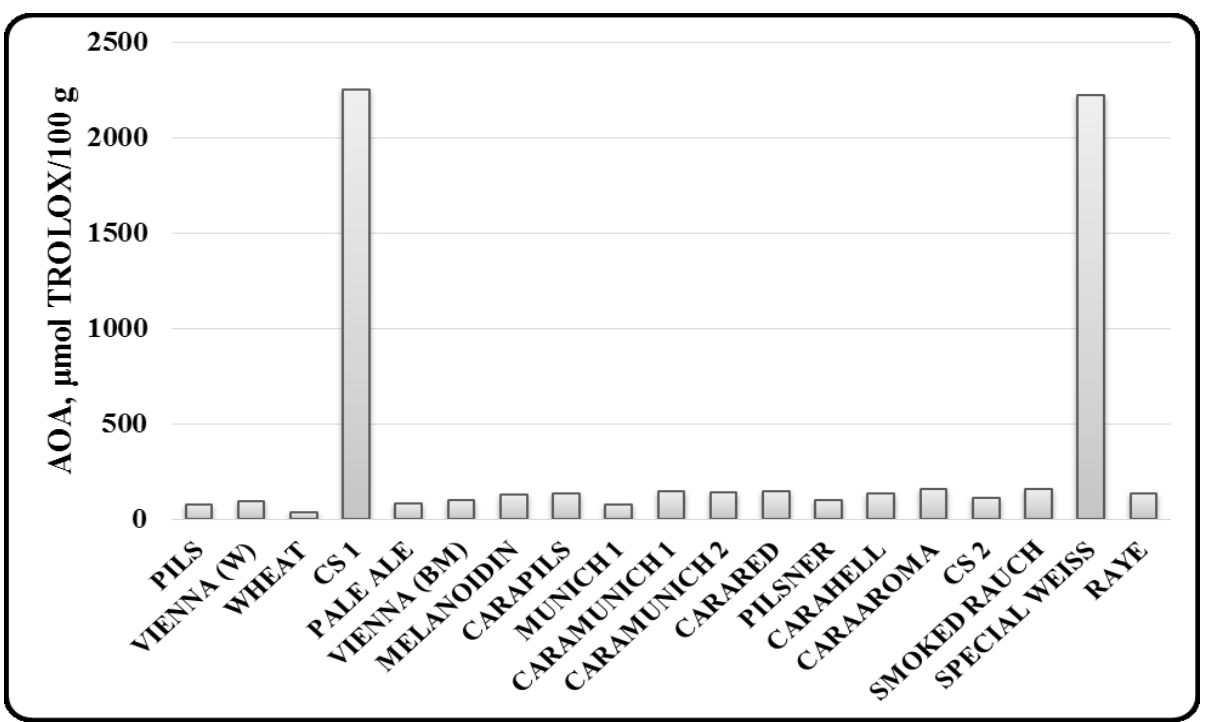

Figure 2. Antioxidant activity (AOA) of brewers' spent grains from different malt types according to the scavenging of the DPPH radical

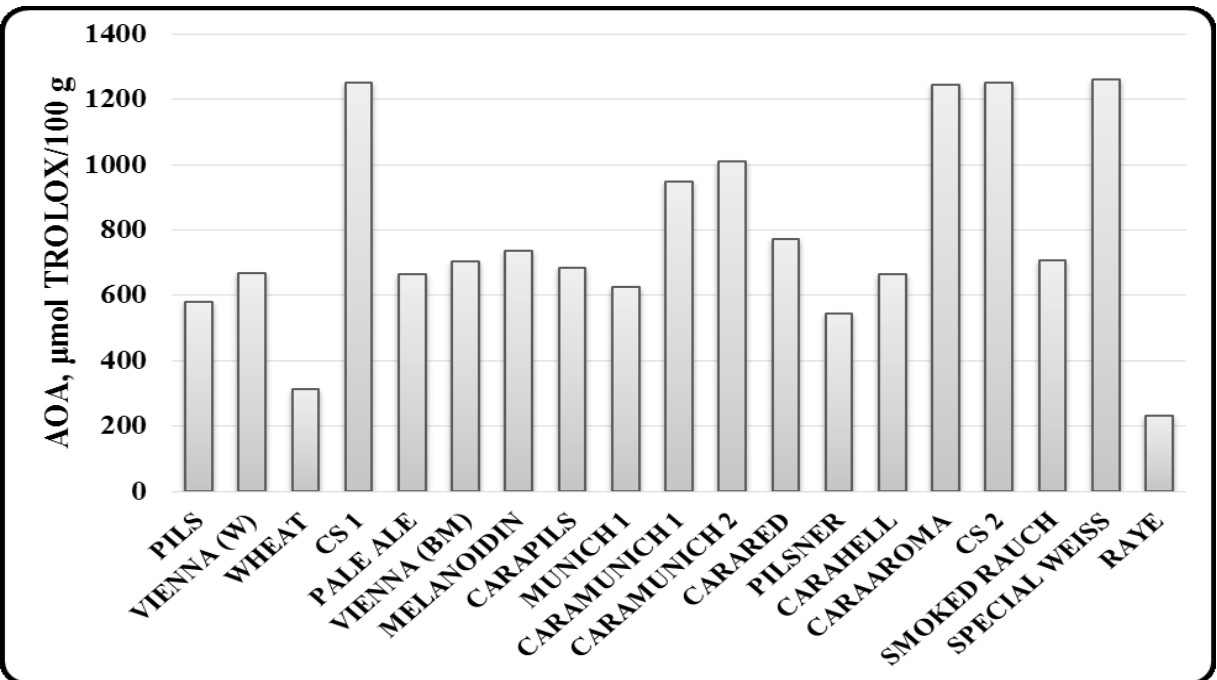

Figure 3. Antioxidant activity (AOA) of brewers' spent grains from different malt types according to the ferric reducing ability (FRAP)

Another potential method for the selection of BSG with a high biological value is by modelling the composition of the malt mixture using the methods of mathematical statistics. This method is applicable to the modeling of the composition of both the malt mixture and the wort. Data related to this can be found in the work of Shopska et al., 2019. In this case, mixture modeling methods that are very effective in determining the proportions of the mixture components can be used (Buruk Sahin et al., 2016).
In the present study, the simplex-centroid method with and without constrains had the widest application. The unrestricted method is suitable for the modeling of mixtures with 3 or 4 components. In the case of increasing the number of components, the constraint method is appropriate. One limitation is the fact that the basic malt types must be present in the mixture in larger quantities (usually between $40 \%$ and $60 \%$ of the total malt quantity). 


\section{CONCLUSIONS}

The present study investigated the phenolic content and antioxidant potential of 19 malts and the resulting BSG. The BSG were found to have a high biological value, which makes them suitable for use in functional foods. The data from the study showed that no single model can be developed for the relationship between the BSG phenolic content and antioxidant potential. A conclusion can be drawn that there is a correlation between the malt color (the degree of kilning) and the antioxidant potential of the malts and their bran. At the end of the paper, a generalized approach to the modeling of the composition of malt bran and the selection of waste products with a high biological value was presented.

\section{ACKNOWLEDGMENT}

The research has been funded under Project No DM17/7 "Scientific approaches for optimizing the extrusion process for deep processing of raw materials from the brewing industry" of the National Science Fund, Bulgaria.

The authors thank the students Juliette Fristot and Antoine-Peïo Uhart for their work in performing the experiments described in this paper.

\section{REFERENCES}

1. Aliyu, S. and M. Bala, M. (2011). Brewer`s spent grain. A review of its potentials and applications. Afr. J. Biotech. 10 (3): 324-331.

2. Analytica-EBC (2007) European Brewery Convention. Nürnberg: Fachverlag Hans Carl, 5th ed.

3. Benzie, I. and Strain, J. (1996) The Feric reducing ability of plasma (FRAP) as a measure of antioxidant activity. The FRAP assay, Anal. Biochem. 189: 70-76..

4. Brand - Williams, W., Cuvelier, M. and Berst, C. (1995) Use of a free radical method to evaluate antioxidant activity, LWT - Food Sci. Technol. 28: 25-30.

5. Buruk Sahin Y., Aktar Demirtas, E. and Burnak, N. (2016). Mixture design: A review of recent applications in the food industry. Pamukkale Üniversitesi Mühendislik Bilimleri Dergisi., 22(4): 297-304.

6. Carvalho D.O., Gonçalves L.M., Guido L.F. (2016) Overall antioxidant properties of malt and how they are influenced by the individual constituents of barley and the malting process. Comprehensive Reviews in Food Science and Food Safety, vol. 15, pp. 927-943

7. Dvořáková, M., Guido, L. F., Dostálek, P., Skulilová, Z., Moreira, M. M. and Barros, A. A. (2008). Antioxidant properties of free, soluble ester and insoluble-bound phenolic compounds in different barley varieties and corresponding malts. Journal of Institute of Brewing, 114: 27-33.

8. Farcas, M., Tofana, M., Socaci, S., Mudura, E., Scrob, S., Salanta, L. and Muresan, V. (2014) Brewer`s spent grain - A new potential ingredient for functional foods. J. Agroalim. Proc. Technol. 20(2): 137-141.

9. Hassona, H.Z. (1993). High fibre bread containing brewer's spent grains and its effect on lipid metabolism in rats. Die Nahrung., 37: 576-582.

10. Inns, E. L., Buggey, L. A., Booer, C., Nursten, H. E. and Ames, J. M. (2011). Effect of modification of the kilning regimen on levels of free ferulic acid and antioxidant activity in malt. J. Agric. Food Chem. 59(17): 9335-9343.

11. Ivanova, K., Denkova, R., Kostov, G., Petrova, T., Bakalov, I., Ruscova, M., and Penov, N. ( 2017) Extrusion of brewers' spent grains and application in the production of functional food. Characteristics of spent grains and optimization of extrusion. J. Inst. Brew., 123: 544- 552. doi: 10.1002/jib.448.

12. Kalcheva - Karadzhova, K. D., Mihalev, K., Ludneva, D., Shikov, V., Dinkova, R. and Bakalov, I. (2016). Effect of pectolytic enzyme preparation on antioxidant capacity and color characteristic of rose petals extract (Rosa Damascena Mill.), Bulg. Chem. Commun. 48 Special Issue E: 464-467.

13. Magalhães, P. J., Almeida, S. M., Carvalho, A. M., Gonçalves, L. M., Pacheco, J. G. and Cruz, J. M. (2011) Influence of malt on the xanthohumol and isoxanthohumol behavior in pale and dark beers. A micro-scale approach, Food Res. Inter. 44(1): 351-359.

14. Maillard, M. N. and Berset, C. (1995). Evolution of antioxidant activity during kilning. Role of insoluble bound phenolic acids of barley and malt. J J. Agric. Food Chem. 43(7): 1789-1793.

15. McCarthy, A. L., O'Callaghan, Y. C., Connolly, A., Piggott, C., FitzGerald, R. J. and O'Brien N. (2012). Phenolic extracts of brewers' spent grain (BSG) as functional ingredients - Assessment of their DNA 
protective effect against oxidant-induced DNA single strand breaks in U937 cells, Food Chem. 134(2): 641-646.

16. Meneses, N. G. T., Martins, S., Teixeira, J. A. and Mussatto, S. (2013). Influence of extraction solvents on the recovery of antioxidant phenolic compounds from brewer's spent grains, Sep. Purif. Technol. 108: $152-158$.

17. Miranda, M.Z., Grossmann, M. V. E. and Nabeshima, E. H. (1994a). Utilization of brewers' spent grain for the production of snacks with fiber. 1. Physicochemical characteristics. Brazilian Archives of Biology and Technology, 37: 483-493.

18. Miranda, M.Z., Grossmann, M. V. E. and Nabeshima, E. H. (1994b). Utilization of brewer spent grain (BSG) for production of snacks with fiber. 2. Sensory analysis of snacks. Brazilian Archives of Biology and Technology, 37: 9-21.

19. Moreira, M. M., Simone Morais, S., Carvalho, D. O., Barros, A. A., Delerue-Matos, C. and Guido, L. F. (2013). Brewer's spent grain from different types of malt. Evaluation of the antioxidant activity and identification of the major phenolic compounds, Food Res. Inter. 54: 382-388.

20. Mussato, S., Dragone, G. and Roberto, I. C. (2006) Brewers' spent grain. generation, characteristics and potential applications, J. Cereal Sci. 43: 1-14.

21. Mussato, S. and Roberto, I. C. (2006). Chemical characterization and liberation of pentose sugars from brewer's spent grain. J. Chem. Technol. Biotechnol. 81: 268-274.

22. Mussatto, S.I., Rocha, G. and Roberto, I. C. (2008). Hydrogen peroxide bleaching of cellulose pulps obtained from brewer's spent grain. Cellulose, 15: 641-649.

23. Öztürk, S., Özboy, O., Cavidoglu, I. and Köksel, H. (2002). Effects of Brewers' spent grains on the qualityand dietary fibre content of cookies. J. Inst. Brew. 108(1): 23-27.

24. Singleton, V. and Rossi, J. (1963) Colorimetry of total phenolics with phosphomolybdic - phosphotungstic acid reagents, Am. J. Enol. Viticul. 50: 3828-3834.

25. Szwajgier D. (2009). Content of individual phenolic acids in worts and beers and their possible contribution to the radical activity of beer. Journal of Insitute of Brewing. 115: 243-252.

26. Shopska, V., Dzivoderova-Zarcheva, M., Ivanova, K., Nedyalkov, P., Denkova-Kostova, R., Kaneva, M. and Kostov, G. (2019). Phytochemical characteristics of different malts and possibillities for their application in functional beverages. Book of proceedings X International Scientific Agriculture Symposium “AGROSYM 2019”: 785-790.

27. Wannenmacher J., Gastl M., and Becker T. (2018). Phenolic substances in beer: Structural diversity, reactive potential and relevance for brewing process and beer quality. Comprehensive Reviews in Food Science and Food Safety, 17: 953-988. 